\title{
Dynamique des postures de chercheurs-engagés
}

Retours sur la participation dans les politiques de l'eau au Burkina Faso

William's Daré et Jean-Philippe Venot

\section{OpenEdition \\ Journals}

Édition électronique

URL : http://journals.openedition.org/anthropodev/514

DOI : 10.4000/anthropodev.514

ISSN : 2553-1719

\section{Éditeur}

Presses universitaires de Louvain

\section{Édition imprimée}

Date de publication : 1 juillet 2016

Pagination : 149-178

ISBN : 797-10-93476-03-2

ISSN : 2276-2019

\section{Référence électronique}

William's Daré et Jean-Philippe Venot, « Dynamique des postures de chercheurs-engagés »,

Anthropologie \& développement [En ligne], 44 | 2016, mis en ligne le 01 janvier 2017, consulté le 10 décembre 2020. URL : http://journals.openedition.org/anthropodev/514; DOI : https://doi.org/ 10.4000/anthropodev. 514

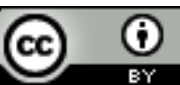

La revue Anthropologie \& développement est mise à disposition selon les termes de la Licence Creative Commons Attribution 4.0 International. 


\title{
Dynamique des postures de chercheurs-engagés
}

\section{Retours sur la participation dans les politiques de l'eau au Burkina Faso}

\author{
William's Daré et Jean-Philippe Venot'
}

\begin{abstract}
Le positionnement des chercheurs et des praticiens de la participation a fait l'objet de nombreuses analyses soulignant la pluralité et la complémentarité des postures adoptées notamment vis-à-vis des jeux de pouvoir. Cet article insiste sur l'aspect dynamique des postures de deux " chercheurs-engagés " ayant piloté un projet de recherche pour le développement, en appui à la politique de gestion intégrée des ressources en eau (GIRE) au Burkina Faso. Définissant l'engagement comme un positionnement à l'interface entre le politique et le scientifique, les chercheurs ont, dès la formulation du projet, explicité leur objectif de favoriser la participation dans l'application de la GIRE et d'utiliser une approche participative pour ce faire. Adoptant initialement une posture de "neutralité dialogique conditionnelle ", à des fins de légitimation vis-à-vis des décideurs politiques burkinabè et de pérennisation de leurs résultats de recherche, les chercheurs ont construit le projet avec ces derniers dans le cadre d'une démarche de modélisation d'accompagnement. Face aux forts différentiels de pouvoir dans la situation d'action, bloquant toute contribution des acteurs locaux à la prise de décision sur l'eau, les chercheurs ont glissé vers une posture de "non-neutralité post-normale »: l'objectif premier a alors
\end{abstract}

\footnotetext{
${ }^{1}$ Nous remercions les deux référents anonymes qui ont permis d'améliorer sensiblement la qualité de cet article. Ce travail a été réalisé dans le cadre du projet CPWF V4, financé par le programme Water, Land and Ecosystems du CGIAR.
} 
été de renforcer la place et le rôle des acteurs locaux dans les dispositifs participatifs afin qu'ils se réapproprient les arènes de concertation créées par le législateur. Les évolutions de postures s'expliquent à la fois par les changements observés dans la situation d'action, la compréhension que les chercheurs en ont et la volonté de préserver leur éthique d'intervention. Si, d'un point de vue heuristique, l'engagement $d u$ chercheur pour la participation dans la gestion des ressources naturelles permet de nourrir une réflexion critique sur l'action publique, il est difficile d'adopter cette posture dans le cadre de projets de recherche pour le développement et dans un contexte fortement structuré par l'aide publique au développement.

The roles, place and influence that researchers and practitioners may have in the way participatory approaches unfold has long been documented. Such analysis notably stress that researchers' stances towards the issue of power may vary significantly. This article do-cuments how the positioning of two "engaged researchers" coordinating a research for development project aimed at supporting Integrated Water Resources Management (IWRM) policies in Burkina Faso have evolved with time. Defining engagement as being at the interface between research and policy, the researchers have made explicit that their objective was to enhance participation in policy implementation and to follow participatory approaches to do so, right from the start of the project. They adopted a stance of "conditional dialogical neutrality" in order to build their legitimacy towards Burkinabe policymakers and ensure the sustainability of their activities, shaping projects activities together with the later and following the precepts of the Companion Modeling approach. How-ever, whereas participation is presented as one of the pillars of IWRM, the administration in charge of piloting the policy proved unable or unwilling to take field realities into account and to create room for natural resources users so that the latter could participate in a meaningful and proactive way. This situation led the resear-chers to progressively shift towards a stance of "post-normal non neutrality", whereby they explicitly 
aimed at strengthening the role and place of local actors in the arenas of participation set up by po-licy makers. Evolution in the positioning of researchers find their roots in the changes observed in the action-space, the understanding researchers have of it, and their willingness to maintain the principles of their intervention. Heuristically, engagement towards the promotion of participation in natural resources management allows for a critical analysis of public action. However, it appears difficult to adopt such a stance in the framework of research for deve-lopment projects and in a context strongly influenced by public development aid practices and networks.

\section{Introduction}

Les interactions entre sciences et sociétés ont évolué non seulement en termes de modes de production de connaissances mais aussi par rapport à la nature même des connaissances produites. La séparation entre sciences et sociétés n'est plus et l'image du chercheur dans sa tour de verre est fortement écornée. Le passage d'un modèle de confinement à un modèle de recherche distribuée (Vinck, 2007) montre l'évolution des modes de production de connaissances et les interpénétrations sans cesse plus fortes entre sciences et sociétés. Cette évolution des interactions se traduit à l'échelle des institutions de recherche mais aussi à l'échelle des chercheurs. Ainsi, de plus en plus de chercheurs en sciences sociales revendiquent une posture de "chercheurengagé " quant à leurs pratiques de recherche et à l'usage qui peut être fait des connaissances produites. Adopter une posture de chercheur-engagé c'est donc assurer un transfert entre le champ scientifique dans lequel le chercheur a construit sa légitimité et le champ politique dans lequel il exprime une position publique.

La relation entre ces deux champs, scientifique et politique, n'est pas univoque. Toute recherche engagée adopte une position politique et est influencée par cette position politique, elle-même sous-tendue par une vision de la place du scientifique dans la Cité. Pour le chercheur, le choix de l'objet de recherche, le corpus 
théorique mobilisé, les méthodes développées, les énoncés scientifiques produits sont liés à des valeurs morales, philosophiques, sociales ou politiques forgées notamment au cours de son histoire de vie. Le chercheur-engagé doit être conscient de ses choix politiques et des implications de sa posture sur sa production scientifique.

Ainsi, développer des recherches participatives pour la gestion des ressources naturelles dans les pays du Sud n'est pas neutre. Comme le souligne d'Aquino (2002:4), "tout programme de développement apporte son idéologie politique implicite "; cela vaut également pour des projets de recherche en faveur du développement, comme celui que nous coordonnions et qui sert de support à cet article. Dans l'élaboration et la conduite de ce projet de recherche, nous avons ainsi revendiqué une posture visant à (1) promouvoir la participation des acteurs locaux dans les politiques de l'eau au Burkina Faso et (2) montrer l'intérêt qu'une telle implication pouvait avoir auprès des décideurs des niveaux régionaux et nationaux. Cela nous a notamment amenés à concevoir et porter une démarche participative comportant un objectif explicite de changement social : améliorer la participation des usagers dans la prise de décision publique sur la gestion des ressources en eau.

Engagement du chercheur et rigueur des connaissances produites

L'engagement du chercheur a longtemps fait l'objet d'une suspicion quant à la rigueur des données produites. Cette suspicion, qui renvoie au débat sur la neutralité axiologique du chercheur en sciences sociales, est diversement débattue selon les disciplines.

Ainsi, en anthropologie, la critique d'une posture de neutralité axiologique peut paraître dépassée tant la relation au terrain est consubstantielle de la discipline (Fassin, 1999; Copans, 2007; Malinowski, 1989). En anthropologie du développement, ce positionnement critique est d'autant plus crucial que l'idée de 
changement social est au cœur de ce courant théorique (Vidal, 2011 ; Olivier de Sardan, 2001). Ainsi, l'idée que l'anthropologue peut à la fois être producteur de connaissances scientifiquement fondées sur une rigueur méthodologique et engagé a largement été débattue (Olivier de Sardan, 2004 et 2000). Le séminaire de l'APAD "Engager l'anthropologie pour le développement et le changement social » tenu en 2010 a permis d'identifier plusieurs dimensions de l'engagement d'anthropologues : choix du terrain et des objets de recherche; implication dans des projets de développement ou des politiques; participation dans le débat public (Hagberg et Ouattara, 2012).

En sociologie classique, les positivistes ont longtemps considéré que le sociologue devait adopter une posture neutre afin de ne pas influencer les faits sociaux qu'il décrivait. Mais, alors que cette position dominante s'imposait en sociologie, la place de la subjectivité dans les analyses de Durkheim ou de Weber était laissée de côté (de Gaulejac et al., 2007). Depuis, une sociologie qualitative s'est développée, s'appuyant sur des études de terrain et empruntant largement ses outils et réflexions à l'anthropologie, notamment quant à la position du chercheur vis-à-vis de son terrain d'étude (de Gaulejac et al., 2007) ou à la scientificité des données ainsi produites (Demeulenaere, 2012). De plus, les sociologies marxistes (Bourdieu, Touraine) développées ont largement milité pour un engagement du sociologue dans l'arène politique. En géographie, ces réflexions restent limitées (d'Aquino, 2002; Barnaud, 2013).

Pour de nombreux auteurs, l'essentiel est de se donner les moyens d'analyser rigoureusement en quoi la subjectivité du chercheur influence les données produites. Le chercheur en sciences sociales qui développe des recherches qualitatives, qui interagit avec l'Autre en pratiquant l'immersion, l'observation participante ou en réalisant des entretiens, devient " une partie prenante du monde social qu'il étudie et ne peut prétendre à une position de pure extériorité " (de Gaulejac et al., 2007 : 14). Quelle que soit la grille d'analyse adoptée - implication vs explicitation (Olivier de Sardan, 2000), implication vs distanciation (Ardoino, 
1990), ou distanciation vs engagement (Fassin, 1999) -, l'important est de pouvoir expliciter la trajectoire personnelle du chercheur sur le terrain, sa relation avec son objet de recherche ainsi que ses interactions avec les acteurs. Cela doit permettre de mieux saisir la qualité du matériel collecté et des analyses produites. L'enjeu est de trouver une voie méthodologique visant à l'objectivité sans neutraliser ou ignorer la subjectivité du chercheur. La subjectivité est en effet un élément essentiel de l'engagement du chercheur auprès de tel ou tel type d'acteurs, en faveur de telle ou telle cause et joue un rôle central dans le processus de production des connaissances.

Partageant le point de vue qui sous-tend ce numéro spécial d'Anthropologie \& Développement, à savoir l'impossible neutralité dans la recherche en sciences sociales, nous revendiquons aussi qu'engagement et recherche, loin d'être antinomiques, se renforcent mutuellement. Appliquée à des enjeux socioenvironnementaux, cette posture nous conduit à expliciter et revendiquer un double engagement, tant éthique que méthodologique. Dans une première partie, nous présentons le contexte de l'étude, à savoir un projet de recherche pour le développement conduit au Burkina Faso en appui à la politique de gestion intégrée des ressources en eau de ce pays, et les étapes de la démarche participative adoptée dans ce projet. Ensuite, nous présentons les deux cadres d'analyse mobilisés afin de porter un regard réflexif sur le projet et notre posture de chercheurs et porteurs du projet. Dans la troisième partie nous analysons comment ces postures ont évolué au cours du projet tant en termes d'accompagnement des acteurs que de positionnement vis-à-vis des jeux de pouvoir inhérents à tout contexte social. Une courte conclusion revient sur ce qu'un tel regard réflexif permet d'identifier et de comprendre des dynamiques d'une " rechercheengagée » pour le développement. 
Contexte : exemple d'un projet de recherche pour le développement

Les travaux présentés ici ont été réalisés dans le cadre d'un projet de recherche pour le développement intitulé « CPWF V4 - Gestion des sous-bassins et gouvernance des eaux de pluie et des petits barrages ", conduit de novembre 2010 à décembre 2013 dans le cadre du Challenge Program for Water and Food (CPWF) ${ }^{2}$. Ce projet, largement conçu et animé par les auteurs de cet article, en collaboration avec des décideurs nationaux du secteur de l'eau, avait pour objectifs d'accompagner les politiques de gestion intégrée des ressources en eau (GIRE) au Burkina Faso et au Ghana et d'interroger les processus et hypothèses qui sous-tendent leur élaboration et mise en place à diverses échelles (seuls les travaux réalisés au Burkina Faso sont discutés ici). Ainsi, sur la base de leurs expériences préalables, les animateurs du projet ont adopté une démarche participative appelée modélisation d'accompagnement (ComMod, 2005), visant à favoriser les interactions entre acteurs de différents niveaux de prise de décision.

\section{Principes de la GIRE et application au Burkina Faso}

Les ressources en eau au Burkina Faso font l'objet d'une double pression : d'une part, une augmentation de la demande en eau liée à la croissance démographique et économique du pays; d'autre part, une dégradation qualitative due à la croissance d'activités anthropiques associée à une diminution quantitative due au changement du régime des pluies et à une plus faible recharge des nappes phréatiques. Dès 1998, le Burkina Faso a été l'un des premiers pays africains à s'engager dans la voie de la GIRE pour relever ces défis.

La GIRE, devenue la norme des politiques de l'eau à l'échelle internationale, s'appuie sur le triptyque du développement durable et se décline en trois piliers : le premier, environnemental, établit le

\footnotetext{
${ }^{2}$ Pour plus d'informations sur le CGIAR Challenge Program on Water and Food, voir : waterandfood.org
} 
bassin hydrographique comme unité de gestion optimale; le deuxième, socio-institutionnel, érige la participation de tous les acteurs comme principe d'action; le troisième, économique, institue l'eau en bien économique et insiste sur la nécessité de la gérer en tant que tel (GWP, 2000). Ces principes et la mise en œuvre de la GIRE ont fait l'objet de nombreuses critiques, notamment dans le contexte des pays en développement et en ce qui concerne le rôle dévolu aux acteurs locaux dans les prises de décision (Blomquist et Schlager, 2005 ; Ghiotti, 2006 ; Chéné, 2009 ; Molle, 2012).

Au Burkina Faso, plus spécifiquement, diverses institutions ont été mises en place depuis le niveau national jusqu'au niveau local afin de favoriser l'implication des différents acteurs et décideurs concernés par la gestion des ressources en eau. Ainsi, la politique burkinabè de l'eau adoptée en juillet 1998 a conduit à l'élaboration d'un Plan d'action pour la GIRE (le PAGIRE) en 2003 et à la création d'agences de l'eau pour sa mise en œuvre. Les agences de l'eau, dont les espaces de compétence recoupent pour l'essentiel les limites des bassins hydrographiques du territoire national, sont composées d'instances et d'organes parmi lesquels figurent les comités locaux de l'eau (CLE). Ces derniers ont été créés pour servir de cadres de concertation pour les acteurs de l'eau au niveau local, autour de points d'eau ou à l'échelle de sous-bassins versants de quelques milliers de kilomètres carrés (DGRE, 2010). Cherlet et Venot (2013) décrivent le processus d'élaboration de la politique nationale de GIRE; Venot et al. (2014) décrivent sa déclinaison à l'échelle locale. Comme dans d'autres contextes, ces deux études montrent que les dynamiques enclenchées offrent peu d'opportunités en termes de participation effective des acteurs locaux.

\section{Le site d'étude : le CLE de la Bougouriba 7}

Le projet CPWF V4 a été conduit en appui au CLE de la Bougouriba 7, membre de l'agence de l'eau du Mouhoun (AEM), la Bougouriba étant un des affluents de ce dernier. Ce CLE a été créé le 9 août 2010 par un arrêté du gouverneur de la région Sud-Ouest. 
Son espace de compétence est un sous-bassin versant de la Bougouriba dont la superficie est de $2732 \mathrm{~km}^{2}$ et couvre tout ou partie du territoire de onze communes appartenant aux provinces de la Bougouriba, du loba et du Poni dans la région Sud-Ouest du Burkina Faso (figure 1). Ces communes regroupaient près de 400 villages en 2008. Les activités économiques dans la zone sont surtout rurales: l'agriculture irriguée, l'agropastoralisme, la pêche et la transformation du poisson ainsi que l'orpaillage artisanal.

\section{Jeux de pouvoir et GIRE à l'échelle locale}

Par statut, les membres des CLE (comme ceux des agences de l'eau) appartiennent à trois collèges - administration, usagers et collectivités territoriales; la répartition entre collèges n'est pas imposée (DGRE, 2010). Ainsi, l'assemblée générale constitutive du CLE de la Bougouriba 7 s'est tenue avec vingt-sept représentants de l'administration, neuf représentants des collectivités locales et sept représentants des usagers (Walaszek, 2012). De plus, la procédure de création du CLE de la Bougouriba 7 n'a pas été participative, comme le montre Walaszek (2012). Elle a été pilotée par les services techniques du ministère de l'Agriculture qui se sont fortement inspirés du travail réalisé précédemment dans le cadre du programme VREO ${ }^{3}$. Le bureau du CLE Bougouriba 7 est certes aujourd'hui composé de trois membres du collège de l'administration, trois membres du collège des collectivités territoriales et six membres du collège des usagers, mais l'administration occupe les postes principaux de président (le hautcommissaire de la province de la Bougouriba), de secrétaire (le directeur provincial de l'agriculture) et de commissaire aux comptes (le préfet de Dano). Les collectivités territoriales, elles, occupent le poste du trésorier. Le contexte de la GIRE dans la zone d'étude est donc marqué par une forte domination de l'administration vis-à-vis

\footnotetext{
${ }^{3}$ Le programme VREO (Valorisation des Ressources en Eau de l'Ouest du Burkina Faso), piloté par les services régionaux du MAHRH, a notamment appuyé la création et l'accompagnement d'un autre CLE dans l'Ouest du Burkina Faso.
} 
des autres collèges, rendant difficile l'application du principe de participation réelle des usagers.

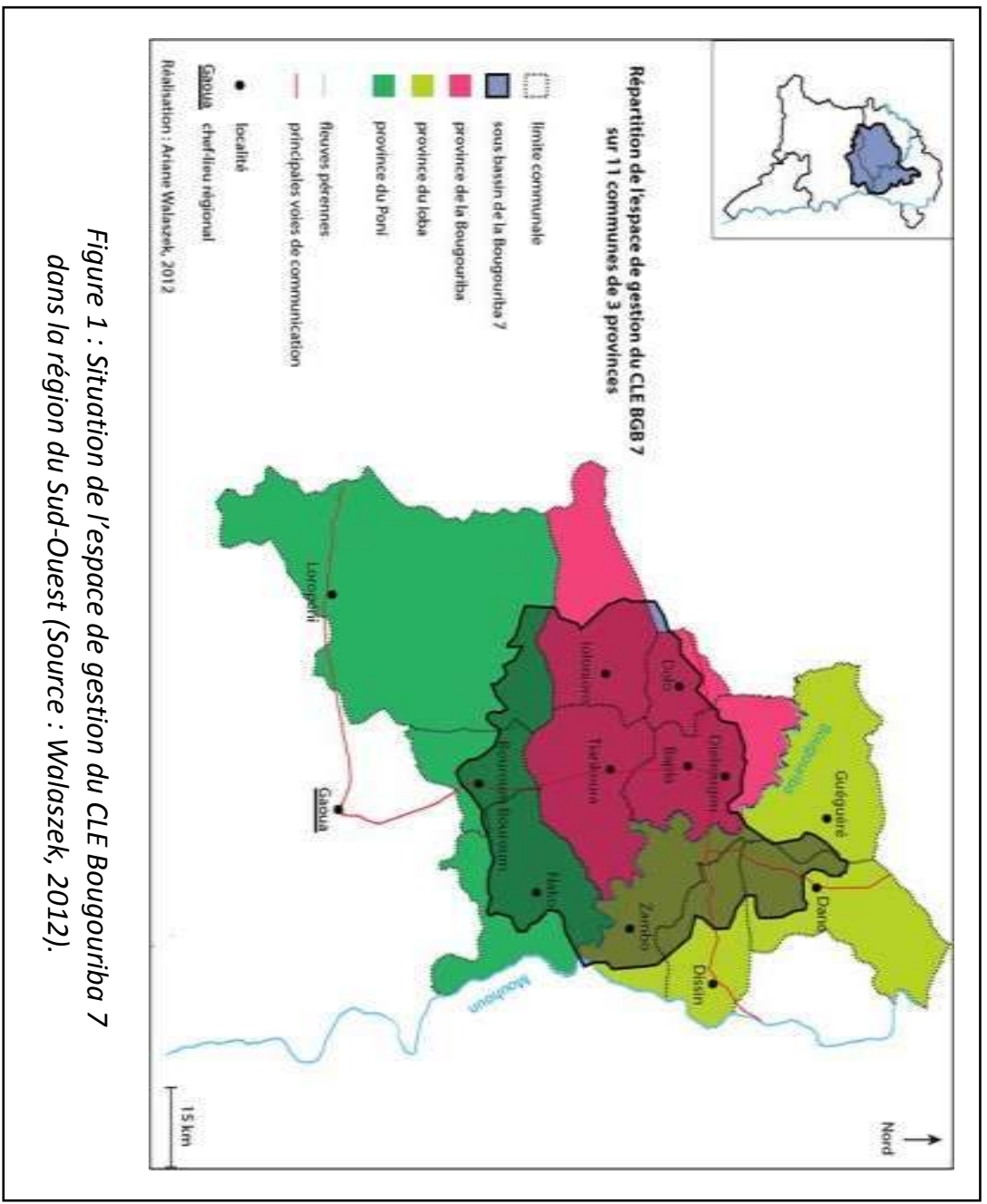




\section{Les étapes du projet de recherche pour le développement}

Le projet de recherche pour le développement que nous avons porté traduit notre posture de "recherche-engagée »: il a été conçu afin de donner aux acteurs locaux la possibilité de contribuer à la prise de décision sur les ressources en eau dans la zone du CLE. Une démarche participative nommée " modélisation d'accompagnement ", dont les moments clés sont présentés dans la figure 2, a été conçue dans ce sens. La modélisation d'accompagnement ou Companion Modelling (ComMod) est une démarche participative appliquée à la gestion des ressources naturelles renouvelables et qui vise deux objectifs principaux : (1) améliorer et partager les connaissances et la compréhension des systèmes de gestion des ressources naturelles et de leurs dynamiques; (2) expliciter les impacts potentiels de propositions visant à résoudre des problèmes identifiés conjointement, ceci se faisant généralement via un accompagnement et une discussion des processus de gestion, de régulation ou de décision (Voinov et Bousquet, 2010 ; ComMod, 2005 ; Etienne, 2010).

Trois principes sous-tendent cette approche :

- Le futur des systèmes sociaux et écologiques n'est pas prédictible car ces systèmes sont complexes, dynamiques et incertains. Ainsi nul ne peut prévoir leur évolution future, un événement pouvant à tout moment en modifier la trajectoire. Aussi, l'accompagnement des processus de gestion et de décision de tels systèmes doit viser à favoriser le développement de capacités leur permettant de s'adapter au mieux à ces évolutions. Ceci se fait notamment en explicitant les multiples interactions et boucles de rétroaction entre les acteurs sociaux et leur environnement.

- La qualité des décisions dépend surtout de la qualité du processus de décision et de celle du dialogue produit. Ainsi, il est plus important d'obtenir un accord sur les règles 
ayant conduit à produire la décision que sur la décision elle-même car, le contexte changeant, une solution trouvée aujourd'hui peut ne plus être valide demain.

Tous les acteurs d'un système social ont leurs propres points de vue sur la réalité, forgés par l'expérience et leurs trajectoires dans l'espace physique et social. Les points de vue sont tous légitimes et peuvent donc tous être exprimés et explicités. Les savoirs élaborés par les scientifiques n'ont pas plus de poids que ceux des autres types d'acteurs : ils ne constituent qu'un point de vue parmi les autres sur la réalité.

La figure 2 représente graphiquement les cinq grandes étapes qui ont jalonné la réalisation du projet CPWF V4 au Burkina Faso, entre mars 2010 et décembre 2013. Quatre de ces étapes sont des "temps forts collectifs" (Etienne, 2010) ayant rassemblé l'équipe du projet ainsi que différents acteurs de la gestion des ressources en eau, comme détaillé ci-dessous.

$1^{\text {ère }}$ étape (Rédaction du projet) : Suite à la publication d'un appel à expression d'intérêt du CPWF, dont l'une des thématiques était la gouvernance des eaux pluviales et des petits barrages dans le bassin de la Volta, le deuxième auteur (chercheur géographe à l'IWMI à l'époque) a contacté six autres chercheurs (en géographie, sociologie, pédologie, hydrologie et modélisation, dont trois membres du collectif ComMod) et le SP-PAGIRE (I'administration en charge de piloter l'implémentation du PAGIRE) pour les associer à la rédaction d'un projet. Entre mars et mai, les échanges ont pris la forme de mails, d'audioconférences et de rencontres bilatérales. En mai, le CPWF a organisé un atelier de rédaction des projets où seuls les deux chercheurs porteurs du projet et le coordinateur du SPPAGIRE, tous trois basés au Burkina, ont été conviés. Ils ont défini ensemble les grandes lignes du projet et formulé la problématique générale comme suit : "Comment limiter l'écart entre théorie et pratiques de la GIRE par le biais 
d'une démarche participative ?" Cette phase s'est terminée en septembre 2010 par la rédaction collective de la version finale du projet. La proposition de projet identifiait clairement la démarche participative proposée. En novembre 2010, un atelier de planification a permis aux huit participants de préciser les méthodes, questions de recherche spécifiques et attentes de chacun. 


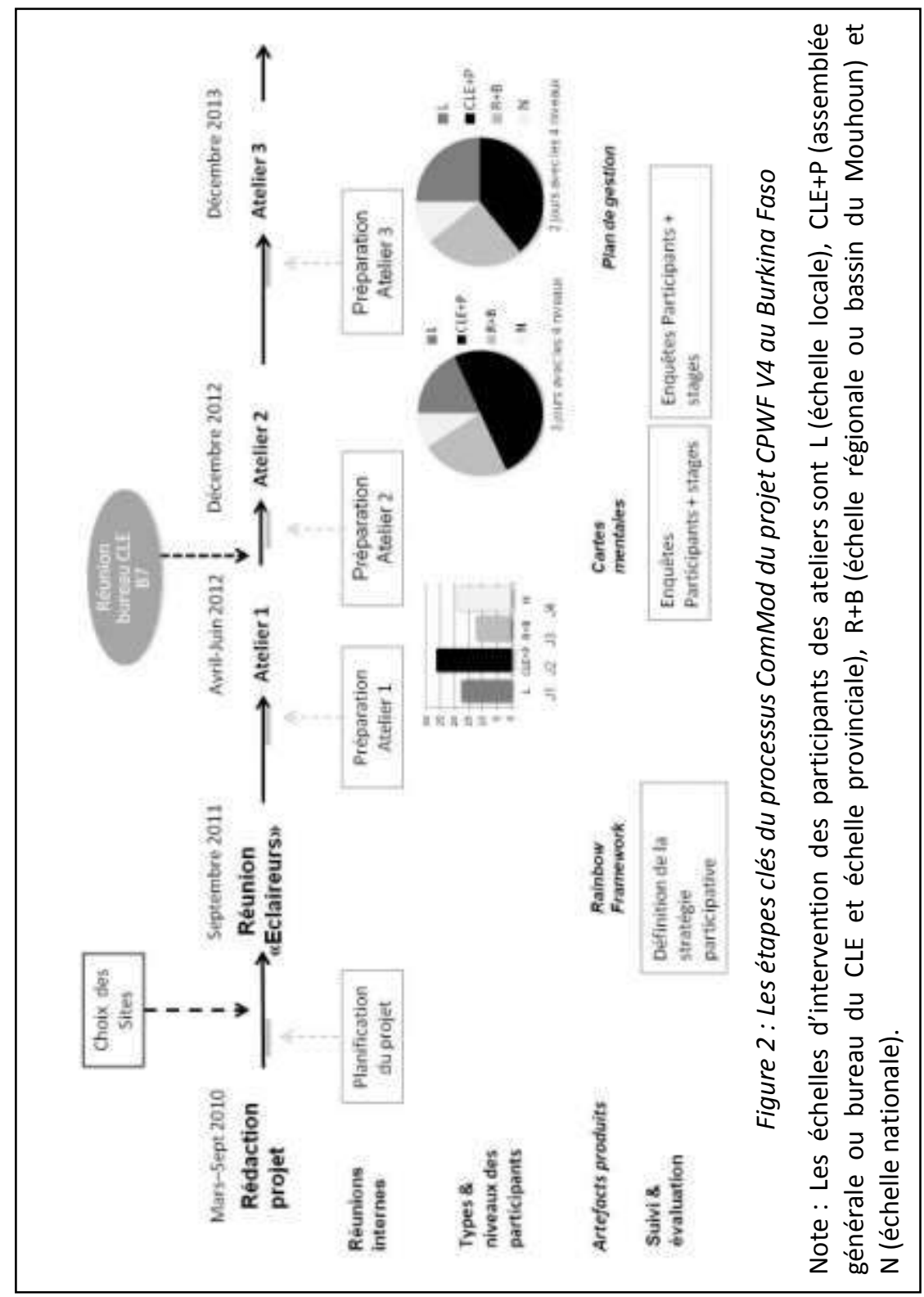


$2^{\mathrm{e}}$ étape (Réunion avec des "éclaireurs ") : Après l'identification (par les huit personnes susmentionnées) du bassin du Mouhoun comme lieu d'exercice du projet notamment en raison du faible nombre d'activités de recherche-développement menées dans ce bassin versant, comparativement à l'autre grand bassin versant du Burkina Faso, le Nakanbé -, nous avons animé un atelier visant à préciser la stratégie participative et à recentrer la problématique du projet. Pour cela, nous avons fait appel à des "éclaireurs ", experts nationaux ou régionaux reconnus par leurs pairs pour leurs travaux sur les enjeux de la GIRE au Burkina Faso et dans la région Sud-Ouest. Les mondes public, privé, scientifique, politique, administratif et financier étaient ainsi représentés au travers de six " éclaireurs " : un consultant indépendant, le président du conseil régional du Sud-Ouest, un chercheur lié au Millenium Challenge Account qui finançait la mise en place d'une dizaine de CLE, le directeur de l'AEM, la responsable de la direction de la Législation et du Suivi des organismes de gestion des ressources en eau au sein du ministère de I'Eau, et le coordonnateur du SP-PAGIRE. Au cours d'un atelier de trois jours organisé en septembre 2011, nous avons amené ces personnes à nous aider à préciser les objectifs de la démarche participative, les acteurs à impliquer, les compétences dont ces derniers disposaient, les apprentissages qui devaient être ciblés, ainsi que les méthodes ou outils à mobiliser et à quel moment du projet. Les "éclaireurs » ont ainsi confirmé notre hypothèse initiale, à savoir que les CLE faisaient face à de nombreux défis, et ils nous ont exhortés à placer les CLE au cœur de notre démarche participative. Suite à cette réunion, le coordinateur du SP-PAGIRE impliqué dans la formulation du projet, en consultation avec les services déconcentrés du ministère de l'Agriculture de la région Sud-Ouest, a choisi de facto le seul CLE existant officiellement au démarrage du projet dans le bassin du Mouhoun. 
$3^{\mathrm{e}}$ étape (Atelier participatif 1 ) : Nous avons organisé un premier atelier participatif en 2012, durant quatre jours (trois jours en avril à Diébougou, chef-lieu de la province de la Bougouriba, situé dans l'espace de compétence du CLE, et un jour en juin à Ouagadougou) afin que différents acteurs puissent expliciter leurs perceptions respectives du CLE. Un exercice de cartographie mentale a été réalisé avec soixante-douze personnes. Les représentants d'usagers de l'eau, non membres des instances (bureau et assemblée générale) du CLE ont été invités le premier jour. Le deuxième jour, les membres du bureau du CLE et des acteurs intervenant à l'échelle des trois provinces du bassin versant de la Bougouriba 7 ont été conviés. Le troisième jour, ce sont des acteurs de la région et de l'AEM qui ont été impliqués dans les exercices proposés. Le quatrième jour a réuni des acteurs de niveau national. Ces quatre journées ont permis de montrer la diversité des perceptions et compréhensions de ce que pouvaient être les rôles du CLE, des enjeux sur lesquels il devait travailler, des acteurs avec lesquels il devait interagir, des actions qu'il devait mener et du mandat qui devait lui être accordé au sein du paysage institutionnel burkinabè de la GIRE.

$4^{\mathrm{e}}$ étape (Atelier participatif 2 ) : Dès mars 2012, il nous est apparu essentiel d'approfondir notre connaissance des alliances et mésalliances s'étant exprimées lors de la mise en place du CLE, et des différentes perceptions locales du fonctionnement de structures collectives de gestion telles que les comités villageois de développement. Deux stages de quatre à six mois ont été organisés à cet effet. En parallèle, suite à ce premier atelier participatif et indépendamment du projet, le bureau du CLE s'est réuni et a émis le souhait que le projet les accompagne pour définir un plan de gestion (dont, légalement, tout CLE devrait se pourvoir). Un deuxième atelier, regroupant vingt-deux personnes (CLE, provinces, région et $A E M$ ) parmi les soixante-douze précédentes, a donc été réalisé en 
décembre 2012, à Diébougou. Cet atelier avait pour but de préciser l'enjeu principal auquel le CLE faisait face et le mode d'organisation que ces acteurs souhaitaient pour élaborer ce plan de gestion. Une technique d'animation par sélection progressive et collective des enjeux exprimés a permis aux participants d'identifier un enjeu principal partagé : "Opérationnaliser le CLE ». Un groupe de travail composé d'un chercheur, d'un représentant du SP-PAGIRE, d'un représentant de la DGRE, d'un autre de l'AEM et de deux membres du CLE a été mis en place pour piloter le processus d'élaboration du plan de gestion. Au préalable, les personnes présentes lors de l'atelier devaient collecter les doléances des personnes qu'elles représentaient. Les représentants des collectivités territoriales devaient solliciter le président du CLE pour qu'il appuie financièrement les réunions nécessaires. Une proposition rédigée par le groupe de travail devait ensuite être discutée par l'ensemble des acteurs. Cependant, rien de tout cela n'a été fait.

$5^{\mathrm{e}}$ étape (Atelier participatif 3) : En décembre 2013, un troisième atelier a été organisé avec les vingt-deux mêmes participants afin de définir les axes principaux et de rédiger collectivement le plan de gestion du CLE. Avec la technique des cartes mentales, nous avons animé les deux jours de réunion dans le but d'aider les participants à exprimer les enjeux dont le CLE devait se saisir, les actions qu'il pouvait mener, et à identifier les responsables de leur mise en œuvre. Après la validation collective des résultats, le groupe de travail s'est chargé de finaliser le document et de chiffrer les activités identifiées. La validation et les discussions de cette proposition devaient être réalisées lors d'une assemblée générale du CLE prévue après la fin du projet. Une assemblée générale s'est en effet tenue en mai 2015. Le bureau y a été renouvelé mais, selon nos informations, le plan de gestion proposé a été validé sans 
être vraiment débattu entre les quarante-trois personnes présentes lors de l'assemblée générale.

Au final, pendant ces trois années et demie, et en tant que coordinateurs du projet, nous avons effectivement eu des interactions quasi hebdomadaires avec nos interlocuteurs du PAGIRE ; mais les interactions ont été moins fréquentes avec les chercheurs associés ainsi qu'avec les membres du CLE Bougouriba 7 et les autres institutionnels de la GIRE au Burkina Faso. Nous avons également piloté l'organisation et l'animation d'ateliers collectifs, la réalisation d'enquêtes et l'encadrement de stagiaires. Entre novembre 2010 et juillet 2012, environ $75 \%$ de notre temps était dévolu aux activités de ce projet. A partir d'août 2012, l'un de nous a continué à assurer le pilotage du projet ( $75 \%$ de son temps), le second, suite à un changement d'employeur, a été moins impliqué (30\%).

Clarification des postures de chercheurs en sciences sociales au sein de démarches participatives

\section{Positionnement des chercheurs au sein de démarches participatives}

Depuis le milieu des années 1990, dans le domaine de la gestion des ressources naturelles, la participation est devenue un leitmotiv des projets de recherche pour le développement (Albaladejo et Casabianca, 1997; Cooke et Kothari, 2001). Les démarches participatives ont cependant fait l'objet de nombreuses critiques. Certains auteurs constatent ainsi que nombre de démarches participatives ne prennent pas en compte les jeux de pouvoir des contextes sociopolitiques dans lesquels elles sont mises en place (Lavigne Delville et Sellamna, 2000 ; Ribot et al., 2006 ; Bodin et Crona, 2008 ; Cooke et Kothari, 2001 ; Barnaud, 2013). Ils critiquent donc leur capacité à créer les conditions d'une réelle implication de l'ensemble des acteurs. Pire encore, ne pas avoir conscience des jeux de pouvoir en place pourrait aussi aboutir à un renforcement de certaines positions dominantes, allant donc à l'encontre de 
l'objectif d'émancipation libératrice souvent associé à la participation. Pris dans les activités routinières des projets participatifs (Liu, 1997), les porteurs de ces derniers ne prennent que rarement le temps de la réflexion, d'autant plus que les outils ou grilles d'analyse permettant de conduire un tel exercice d'autoréflexion sont rares.

Barnaud et al. (2015) soulignent ainsi le manque de méthodes pour questionner le positionnement des porteurs de démarches participatives vis-à-vis des jeux de pouvoir. Ces auteurs distinguent deux approches principales de la participation. D'un côté, les approches dialogiques pour lesquelles la communication entre les parties prenantes permet de mieux se comprendre et favorise les apprentissages qui réduisent les asymétries de pouvoir. De l'autre, les approches critiques où les porteurs, actifs et stratégiques, visent à réduire les inégalités de pouvoir entre les parties prenantes comme elles ont été exprimées lors des ateliers participatifs. En enquêtant cinquante et un praticiens de démarches participatives, chercheurs ou non, ils identifient cinq postures types : la neutralité de type laisser faire, la neutralité dialogique inconditionnelle, la neutralité dialogique conditionnelle, la non-neutralité post-normale, et la non-neutralité stratégique (Barnaud et al., 2015). L'intérêt méthodologique de ce travail est manifeste mais il donne une vision statique de la posture du chercheur-praticien alors que ce dernier peut la modifier et l'adapter en fonction de l'évolution du contexte d'intervention et de la compréhension qu'il peut en avoir.

Beauvais (2007) comble cette limite en proposant une analyse dynamique et réflexive de la posture du chercheur-accompagnateur qui appuie une institution dans la définition de son projet de formation. S'interrogeant sur la capacité du chercheuraccompagnateur à viser l'autonomisation de l'Autre, l'auteure souligne la pluralité et la singularité de la posture de chercheur et identifie trois temps forts qui marquent l'intervention de ce dernier : la compréhension, l'agir, et la retenue. Le temps de la compréhension est celui d'un double échange où le recueil d'informations permet de mieux comprendre l'institution accompagnée et où le chercheur-accompagnateur donne à 
l'institution des éléments pour le comprendre. "La difficulté essentielle réside (donc) dans la lisibilité des postures" (Beauvais, 2007 : 47), qui doivent être clarifiées : chercheur, consultant ou expert d'un côté; commanditaire, décideur, objet ou sujet de recherche de l'autre. Le temps de l'agir est celui où le chercheur répond à une demande intentionnelle de l'institution accompagnée ou l'aide à gérer une crise. Mais il ne s'agit pas de " faire à la place de ". Ici, ce sont les compétences du chercheur-facilitateur qui sont explicitement sollicitées, que ce soit pour animer une réunion ou conduire des entretiens. Le chercheur passe ainsi d'une posture compréhensive à une posture active. Mais ce changement doit s'appuyer sur une " ligne de conduite » (Goffman, 1974) ${ }^{4}$ cohérente et comprise de l'institution accompagnée. Enfin, le temps de la retenue est celui du " retrait » du chercheur-accompagnateur pour favoriser l'autonomisation de l'Autre dans la conception et la réalisation de son projet. Une telle posture est risquée car l'institution est en demande d'expertise et s'attend à ce que le chercheur-accompagnateur y réponde.

Beauvais (2007) définit ces postures non pas au regard des jeux de pouvoir mais bien en fonction de la nature des interactions entre le chercheur et l'institution accompagnée. Cependant, ces temps et les postures associées ne sont pas exclusifs mais s'expriment de façon plus ou moins forte dans le temps. Cela rend donc la posture du chercheur-accompagnateur risquée car elle n'est pas donnée en soi et doit continuellement s'adapter entre le rôle du chercheur et celui de l'accompagnateur.

4 Bien que n'y faisant pas référence explicitement, ces deux premiers temps de Beauvais renvoient à la règle de la "face " décrite par Goffman comme condition à toute interaction. Le concept de "face " représente " la valeur sociale positive qu'une personne revendique effectivement à travers la ligne d'action que les autres supposent qu'elle a adoptée au cours d'un contact particulier»(Goffman, 1974). L'interaction est fondée sur l'acceptation mutuelle de la " face " et de la " ligne de conduite " de chacun. Cette acceptation permet à chacun de prédire le comportement des autres et de se comporter de manière à susciter un comportement désiré de ceux-ci. 
Analyses réflexives portées sur des processus de modélisation d'accompagnement

Les porteurs de démarches de modélisation d'accompagnement mobilisent diverses techniques de diagnostic multi-acteurs pour analyser le contexte (enquêtes, questionnaires, etc.). Et ils conçoivent, avec ces mêmes acteurs, des outils permettant de simuler différentes options de gestion, de les discuter collectivement et de les tester lors de processus d'apprentissage collaboratif (système multi-agents, jeu de rôles, cartographie participative, etc.). Plusieurs sociologues ont décrit leur positionnement dans des démarches développées en France ou à l'étranger au sein d'équipes pluridisciplinaires constituées à cette occasion.

Dans l'équipe pluridisciplinaire chargée de porter une démarche ComMod sur le thème de la sécurisation foncière au Sénégal et à la Réunion, Daré et al. (2007) montrent la tension entre leurs différents positionnements, endogènes ou exogènes. En effet, ils occupaient à la fois un rôle d'observateur des dynamiques et interactions des membres de l'équipe et un rôle de participant, fournissant les connaissances nécessaires à la compréhension, puis à la modélisation des dynamiques sociales en lien avec les dynamiques écologiques. Conscients de leur engagement, les sociologues revendiquent une posture de non-neutralité où ils participent à la fabrication du social :

"Le sociologue, [...] observateur impliqué [...], participe au processus de dévoilement, à la circulation de l'information entre

différents protagonistes et, ce faisant, il contribue à la modification des rapports sociaux entre les individus et à propos de leur environnement » (Daré et al., 2007 : 113).

Charles et al. (2008) ont participé, en France, à quatre démarches ComMod visant à la sensibilisation d'acteurs de réserves de biosphère par rapport à la transformation des paysages du fait du développement de l'embroussaillement. Ces auteurs confirment la position d'entre-deux des ethno-sociologues impliqués dans le 
dispositif participatif comme observateurs et producteurs de connaissances. Ils soulignent que le lien entre politique et sciences est relativement flou dans un processus ComMod où l'implication d'acteurs scientifiques se traduit notamment par une interpénétration des sphères politiques et scientifiques. Le chercheur en effet entre dans l'arène politique en se saisissant directement d'une question de société ou en étant mandaté par des acteurs pour traiter cette dernière.

Les démarches ComMod, comme d'autres approches participatives, ont également fait l'objet d'analyses en termes de jeux de pouvoir. Au travers d'une expérience conduite à Ouessant, Chlous-Ducharme et Gourmelon (2008) s'interrogent sur le rôle des outils pour favoriser la participation des citoyens. Certes, les savoirs scientifiques et les autres types de savoirs interviennent dans la conception des outils et, ensuite, dans leur utilisation ludique, mais les acteurs non scientifiques restent peu impliqués dans la définition première de la problématique. Or c'est une étape fondamentale de la démocratie dialogique. Aussi, même si les itérations du processus ComMod permettent de prendre en compte une grande pluralité de savoirs, les auteurs questionnent le risque que ces approches puissent reproduire des rapports de domination préexistants, notamment entre scientifiques, experts et acteurs locaux. La section qui suit explicite comment notre engagement a pu évoluer dans le temps du projet, non seulement en lien avec les jeux de pouvoir du contexte social dans lequel nous nous placions, mais aussi vis-à-vis des savoirs et des représentations échangés.

\section{Analyse réflexive de chercheurs engagés dans l'action}

Dans cette section nous analysons les évolutions de nos postures et formes d'engagement, en lien notamment avec notre compréhension des jeux de pouvoir et des savoirs et représentations échangés au cours du projet. Nous avons ainsi été tour à tour initiateurs de la démarche, promoteurs de la constitution d'un groupe d'acteurs à impliquer dans celle-ci, 
organisateurs et facilitateurs de différents ateliers participatifs, garants d'un certain équilibre dans les échanges dans un contexte de différentiels de pouvoir importants, porte-parole des points de vue et représentations des différents participants, observateurs des interactions, transcripteurs de savoirs, diffuseurs de connaissances, enquêteurs, tout en déclinant une réflexion de recherche sur les politiques publiques au cours des trois ans et demi qu'a duré le projet.

\section{En attendant le premier atelier participatif : une posture} de neutralité dialogique conditionnelle pour comprendre

Chercheurs et représentants du SP-PAGIRE ont rédigé le projet ensemble. Les autres acteurs, et notamment les acteurs locaux du CLE de la Bougouriba 7, n'ont pas été impliqués dans cette première phase. II y a tout d'abord des raisons pratiques à cela: nous n'avions pas alors identifié la zone sur laquelle porteraient nos activités de recherche-engagée. De plus, à ce stade, notre objectif était à la fois de conduire une analyse critique de la politique de l'eau au Burkina Faso et d'accompagner sa mise en œuvre afin que les résultats de la démarche puissent être pérennisés au-delà du temps du projet et dans d'autres régions que celle choisie. L'implication du SP-PAGIRE dès cette première phase de définition de la problématique était pour nous essentielle. En effet, si cet acteur adhérait aux principes de la démarche ComMod, il nous semblait être le plus à même d'inscrire cette démarche dans son agenda (politique) et d'en assurer non seulement la continuité auprès du CLE avec lequel la démarche aurait été développée mais aussi la diffusion auprès des autres CLE et agences de l'eau qu'il appuie.

A ce stade, il s'agissait pour nous, chercheurs, de bien nous faire comprendre auprès de notre interlocuteur privilégié mais également de saisir au mieux ses enjeux et ses attentes vis-à-vis de sa participation dans le projet. Comme l'indique la formulation de la problématique, nous avons aussi fait le choix de mettre en avant les enjeux d' " accompagnement" plutôt que ceux d' " analyse critique » qui caractérisent la recherche en sciences sociales sur la 
GIRE. Alors que dans nos analyses nous pointions du doigt une approche descendante de la GIRE à l'échelle nationale et d'importants différentiels de pouvoir, nous avons adopté une "posture dialogique conditionnelle» de neutralité car elle était pour nous la seule qui faisait sens pour construire notre légitimité d'intervention.

L'atelier avec les éclaireurs nous a permis d'améliorer notre connaissance du système sociopolitique et écologique de la GIRE au Burkina Faso, ainsi que des jeux de pouvoir inhérents, et de nous focaliser sur la région Sud-Ouest. Sur la base du dialogue que nous avons initié avec un nombre limité d'experts et de représentants de l'administration de l'eau au Burkina Faso, les difficultés de fonctionnement des CLE (identifiées également par d'autres chercheurs) nous sont apparues comme l'enjeu principal pour fédérer les énergies des acteurs à différents niveaux. La procédure de dialogue que nous proposions au travers de la démarche ComMod, les outils que nous maîtrisions et les principes que nous revendiquions, concouraient à la construction de notre légitimité.

Difficile équilibre entre action et retenue : une posture de non-neutralité post-normale

Le premier atelier visait à rendre explicite les points de vue des acteurs des différents niveaux de gestion et de décision, depuis l'échelle nationale jusqu'à l'échelle locale. Dans ce but nous avons créé les conditions pour que chaque partie puisse s'exprimer librement - l'animation via l'élabo-ration de cartes mentales permettant aux participants de donner leur avis de façon quasi méthodique et systématique, sans influence d'un tiers. A ce stade, il ne s'agissait pas d'accompagner le CLE de la Bougouriba 7 mais bien de le repositionner dans le paysage des acteurs de la GIRE : pas d'action directe avec le CLE si ce n'était de faire expliciter et partager la diversité des points de vue des acteurs quant aux rôles, enjeux à saisir, responsabilités à endosser, réseau d'acteurs à mobiliser et actions à réaliser. Il ne s'agissait pas d'agir mais de donner à comprendre. Toutefois, ce faisant, en animant l'atelier, nous entrions tout de même dans l'action, accompagnant de fait le 
SP-PAGIRE dans l'analyse mais aussi la mise en œuvre de sa propre politique, par le biais d'activités perçues par nombre d'acteurs comme un " renforcement des capacités » du CLE. Ici, il ne s'agissait pas de se substituer au SP-PAGIRE mais de trouver un équilibre entre compréhension, action et retenue.

Notre posture de chercheur-engagé devait être en phase avec cette recherche d'équilibre. Nous avons ainsi adopté une posture de " non-neutralité post-normale » en créant les conditions pour que les acteurs locaux et membres du CLE puissent s'exprimer au même titre que les acteurs des autres niveaux, une opportunité qu'ils avaient rarement eue auparavant. En invitant des représentants des usagers à évoquer leurs propres activités et identifier leurs liens potentiels avec cette entité alors inconnue que constituait le CLE, nous avons de fait initié une dynamique où les plus vulnérables pouvaient s'exprimer davantage: pas de dialogue direct entre les niveaux lors de ce premier atelier participatif car nous considérions que cela risquait d'inhiber ces derniers, mais un réel engagement à faire s'exprimer tout un chacun, qu'il soit membre ou non du collège administratif dominant la GIRE. Le choix de la technique d'animation (les cartes mentales) a alors joué un rôle fondamental dans la construction de notre légitimité en tant que chercheurs venus stimuler le « réveil » du CLE.

\section{Un plongeon dans l'action : l'animation de la rédaction collective du plan de gestion du CLE}

Suite au premier atelier participatif, le bureau du CLE s'est réuni et a demandé à ce que les porteurs du projet les accompagnent dans la rédaction de leur plan de gestion. Nous sommes ainsi entrés dans une phase " pure " d'action auprès des vingt-deux participants venant des quatre niveaux précédemment identifiés (du local au national). Bien que résolument inscrit dans l'action, le deuxième atelier participatif avait pour ambition de ne servir que de maïeutique à un projet qui devait être porté par les acteurs de l'atelier - et non par les porteurs du projet. L'organisation des échanges en petits sous-groupes homogènes (trois à quatre personnes) et une technique d'animation visant à la hiérarchisation 
progressive des enjeux de chacun ont visé principalement à organiser les réflexions des participants, faciliter les débats, favoriser la prise de position et acter les décisions prises. Nous n'avons outrepassé notre rôle de facilitateur que lorsqu'il s'est agi de définir les conditions et l'organisation pour la rédaction de ce plan de gestion. En effet, ayant comme point de référence la façon dont de nombreux projets de développement se déroulent, certains acteurs souhaitaient faire appel à des compétences extérieures, comme celles de consultants, pour rédiger ce plan. Nous leur avons alors rappelé les contraintes d'un tel choix pour un CLE qui n'a aucunes ressources financières propres, ainsi que le risque de se voir déposséder de leur propre avenir s'ils n'étaient pas suffisamment actifs dans la production de ce document. Sur cette base, un groupe de travail - dominé par l'administration malgré notre souhait affiché de renforcer le poids des usagers au sein du CLE - a été formé pour préparer ce plan de gestion.

Nous avons ensuite animé le troisième atelier, au cours duquel les axes du plan de gestion et les enjeux de la zone ont été définis par ce même collectif. Malgré les engagements de chacun lors de l'atelier précédent, aucune information issue de la base n'avait été formellement collectée et ce bien que ce troisième atelier se soit tenu un an après le deuxième. Outre des aspects pratiques d'organisation, le fait de conduire un atelier un an après le deuxième avait pour but de "tester " le niveau d'appropriation de la démarche par l'ensemble des acteurs. Cette stratégie s'est heurtée à l'absence de ressources financières propres du CLE dans un contexte où les per diem sont devenus indissociables de toute activité de développement portée par des tiers, et à un manque de dynamisme et de volonté de l'administration locale peu encline à s'engager pour dynamiser le CLE. Ne pouvant pas repousser l'atelier (le financement du projet se terminant en décembre 2013) et n'ayant pas les moyens de faire émerger une expression des acteurs à la base, nous avons choisi malgré tout de tenir ce dernier atelier. Conscients des jeux de pouvoir dans lesquels se plaçait notre intervention, et notamment de l'importance prise par l'administration et les services techniques de l'eau et du frein que 
cela pouvait constituer, et forts de la légitimité d'avoir été sollicités par le CLE, nous avons continué d'adopter une posture de "nonneutralité post-normale ». Lors de cet atelier 3 , nous avons mobilisé des outils d'animation permettant l'expression de chacun des participants: représentants d'usagers et des collectivités locales, provinciales et régionales, membres du bureau du CLE, de l'AEM, du SP-PAGIRE et de la DGRE. Les usagers directs et certains maires ont fait part de leur mécontentement quant à l'inactivité du président et du secrétaire du CLE alors que des pollutions de l'eau, d'origines diverses, sont déjà constatées, constituant de véritables dangers sanitaires et ayant des conséquences économiques graves pour certains usagers (avec la mortalité accrue de poissons destinés à la pêche puis à la vente sur les marchés locaux). Les axes principaux qui structurent le plan de gestion du CLE ont été identifiés collectivement, mais la rédaction finale, incluant une évaluation du coût financier des différentes activités, a encore été réalisée par le SP-PAGIRE, la DGRE et un membre du CLE. Bien que ce même groupe ait été très peu actif entre les ateliers 2 et 3 , les participants ont jugé que les premiers, issus de l'administration, étaient les seuls compétents techniquement (disposant d'un ordinateur et capables de chiffrer les activités) mais qu'un membre du CLE était nécessaire pour s'assurer du respect des décisions collectives prises. Nous avons cependant vérifié la fidélité du document final vis-à-vis des décisions prises par le collectif. Comme nous l'avons décrit plus haut, ce document final a été laissé de côté pendant seize mois, ce qui illustre les limites de notre stratégie visant à pérenniser à l'échelle nationale les actions et les résultats obtenus localement tout en construisant notre légitimité vis-à-vis de l'administration en charge du pilotage des politiques publiques. Une telle stratégie d'intervention nous a empêchés, de fait, de questionner les jeux de pouvoir et d'influence de façon trop frontale!

\section{Conclusion}

La présente contribution porte un regard réflexif sur les dynamiques de nos postures de "chercheurs-engagés " au sein d'un projet de 
recherche participative pour le développement, en appui à la politique de GIRE au Burkina Faso. A l'interface entre le " politique » et le "scientifique ", notre positionnement a évolué avec le temps du projet, en lien à la fois avec l'évolution de la situation d'action et celle de notre éthique d'intervention. Cette dernière se basait sur un postulat - celui de la nécessité d'un dialogue et d'une action collective multi-niveaux pour une gestion durable des ressources naturelles - et un choix - celui d'une démarche participative comme mode de construction et de partage des savoirs. Les simplifications opérées dans la description du projet de recherche que nous avons piloté visaient à rendre notre expérience intelligible mais ne doivent pas occulter la complexité de l'approche réflexive. Ainsi, cette description fait-elle ressortir trois grandes questions qui doivent être posées pour rendre compte de l'évolution de l'engagement du chercheur: auprès de qui s'engage-t-il ? A quelle fin ? Comment se traduit cet engagement dans ses actions de recherche?

Auprès de qui ? Même si la démarche participative mise en place visait pour l'essentiel à un appui aux acteurs locaux pris dans des jeux de pouvoir vis-à-vis des décideurs nationaux, notamment de l'administration burkinabè, les analyses de nos activités de recherche confirment que les jeux sociaux sont complexes et multiformes. Ainsi des jeux de pouvoir existent également : entre usagers directs (maraîchers et orpailleurs vs pêcheurs); entre usagers directs et élus locaux; entre usagers directs et représentants des collectivités locales vis-à-vis des hauts fonctionnaires provinciaux et régionaux. L'évolution de notre posture d'engagement s'explique également par l'amélioration in itinere de notre compréhension de ces jeux de pouvoir complexes afin de rester en phase avec notre éthique d'accompagnement.

A quelle fin? Partant d'une perspective de "l'agir communicationnel » habermassien, notre objectif était de renforcer le dialogue entre les différents acteurs de la GIRE. Ainsi, notre intervention visait à introduire un nouveau format de participation où les acteurs locaux seraient davantage en capacité d'intervenir dans les processus de décision. Pour nous, cet objectif premier 
passait par des objectifs intermédiaires. Tout d'abord, les autres acteurs reconnaissant la nécessité de " renforcer les capacités des acteurs locaux ", notre démarche s'est focalisée sur l'amélioration de la compréhension des enjeux de la GIRE par ceux-ci et la place du CLE et des usagers dans le dispositif institutionnel national. Ensuite, il s'agissait d'accompagner les acteurs locaux afin qu'ils puissent présenter un point de vue argumenté de leur position face aux acteurs des niveaux supérieurs. Mais, mettre les acteurs locaux en position de dialogue n'a de sens que si les autres interlocuteurs sont prêts, eux aussi, à engager le dialogue. Ainsi, afin d'instiller l'idée de ce dialogue entre acteurs locaux et administration, que nous considérions nécessaire, nous sommes aussi intervenus en dehors du cadre du projet stricto sensu auprès du SP-PAGIRE, des agences de l'eau et d'autorités de gestion de bassins transfrontaliers (Niger et Volta). Dans ces arènes, nous exprimions une vision critique de la mise en œuvre de la GIRE au Burkina Faso et proposions qu'une consultation et une participation accrues des usagers des ressources pourraient, peut-être, permettre de trouver des solutions aux difficultés d'application de la politique que tous reconnaissaient.

Comment ? Les contraintes financières et temporelles (rendus de "délivrables ", programmation des activités, partenariat avec l'administration nationale) imposées par un projet de recherche pour le développement vont à l'encontre d'une volonté d'adaptation au temps de nos interlocuteurs, ainsi que d'une posture de retenue permettant aux acteurs de s'approprier au mieux la démarche participative initiée. Ainsi, cette tension entre posture et contexte de mise en place s'est-elle traduite par des lenteurs: un an entre les ateliers 2 et 3 ; trois ateliers participatifs réalisés au lieu des cinq initialement programmés. Sans doute avons-nous péché par excès de confiance en ce qui concerne les dynamiques locales d'appropriation que nous souhaitions faire émerger. Sans doute aurions-nous dû être plus actifs auprès des acteurs locaux qui critiquaient ouvertement la position attentiste des responsables du CLE. Sans doute aurions-nous dû de même être plus actifs auprès du président et du secrétaire du CLE afin de 
stimuler leurs réflexions quant à leur rôle et à celui des usagers et à l'intérêt qu'ils pourraient trouver dans une plus grande autonomisation du CLE. La poursuite de notre engagement au-delà de ce projet afin d'accompagner ces mêmes acteurs dans la mise en œuvre d'une action prévue dans leur plan de gestion, en partenariat avec une structure locale, constitue un prolongement de nos activités pour compenser les difficultés constatées.

Enfin, d'un point de vue heuristique, il nous semble que l'engagement a été pour nous un moyen de nourrir une réflexion critique sur l'action publique que d'autres approches en science sociales, plus distantes, ne permettraient pas. Cela nous a été permis du fait d'une certaine proximité, construite dans le temps, avec les décideurs. Cependant, un tel positionnement et le cadre dans lequel nous l'avons déployé, celui d'un projet de recherche pour le développement, peuvent paraître antinomiques. En effet, l'inscription d'une posture engagée dans un projet de recherche pour le développement limité dans le temps (trois ans et demi) et mobilisant la participation pour induire du changement social est risquée, voire paradoxale. Ainsi, une question demeure, celle de l'adéquation entre notre éthique d'accompagnement et notre engagement dans la Cité. La recherche d'un idéal participatif où les acteurs seraient impliqués dès la définition de la problématique du projet, qui est pourtant l'un des fondements d'une démocratie dialogique, n'est-elle pas illusoire dans un contexte structuré par l'aide publique au développement et dominé par l'administration ?

\section{Bibliographie}

ALBALADEJO C. et CASABIANCA F., 1997, "Eléments pour un débat autour des pratiques de recherche-action ", Etudes Recherches Syst. Agraires Dév., 30 : 127-149.

ARDOINO J., 1990, "Les postures (ou impostures) respectives du chercheur, de l'expert et du consultant ", in ARDOINO J. et BERGER G. (eds), Actes du Colloque Les nouvelles formes de la Recherche en éducation au regard d'une europe en devenir, 
Alençon, Association Francophone Internationale de Recherche Scientifique en Education : 22-30.

BARNAUD C., D'AQUINO P., DARE W., FOURAGE C. et MATHEVET R., 2015, " Dispositifs participatifs et asymétries de pouvoir ", in Actes du colloque du GIS Démocratie et Participation Chercheur.es et acteur.es de la participation, Liaisons dangereuses et relations fructueuses, 29-30 janvier 2015, Paris.

BARNAUD C., 2013, "La participation, une légitimité en question », Natures Sciences Sociétés, 21 : 11p.

BEAUVAIS M., 2007, « Chercheur-Accompagnateur : une posture plurielle et singulière ", Recherches Qualitatives, Hors Série (3) : 44-58.

BLOMQUIST W. et SCHLAGER E., 2005, "Political Pitfalls of Integrated Watershed Management", Society \& Natural Resources, 18(2): 101-117.

BODIN O. et CRONA B., 2008, "Management of Natural Resources at the Community Level: Exploring the Role of Social Capital and Leadership in a Rural Fishing Community", World Development, 36(12) : 2763-2779.

CHARLES M., CHLOUS-DUCHARME F., FAUGERE E. et WINTZ M., 2008, "Science et démocratie dans la gestion de la nature : des ethnosociologues pris dans la modélisation d'accompagnement ", VertigO, 8(2), $17 \mathrm{p}$.

CHENE J.M., 2009, "Introduction - Integrated water resources management: Theory versus practice", Natural Resources Forum, (33) : 2-5.

CHERLET J. et VENOT J.-P., 2013, "Structure and agency: understanding water policy changes in West Africa", Water Policy, 15(3) : 479495.

CHLOUS-DUCHARME F. et GOURMELON F., 2008, " Modélisation et jeu de rôles sur l'île d'Ouessant : questions de sociologie ", Socio-Logos, 3.

COMMOD, 2005, "La modélisation comme outil d'accompagnement ", Natures Sciences Sociétés, 13 : 165-168.

COOKE N. et KOTHARI U., 2001, Participation: The New Tyranny?, London, Zed Books. 
COPANS J., 2007, "Itinéraires de chercheurs: introduction ", Revue Tiers Monde, 191(3) : 489-495.

D'AQUINO P., 2002, "Le territoire entre espace et pouvoir: pour une planification territoriale ascendante ", Espace Géographique, $31(1): 3-22$.

DARE W., FOURAGE C. et GAYE I.D., 2007, "Positionnement des sociologues dans la démarche de modélisation Domino ", Nouvelles Perspectives en Sciences Sociales, 2(2) : 103-126.

DGRE, 2010, "Les Comités locaux de l'eau (CLE) : document guide de conception, création et fonctionnement ", Ouagadougou.

DE GAULEJAC V., HANIQUE F. et ROCHE P., 2007, La sociologie clinique: enjeux théoriques et méthodologiques, Paris, Eres.

DEMEULENAERE P., 2012, " L'interprétation des terrains et le recours à des interprétations théoriques ", L'Année sociologique, 62(1) : 67-91.

ETIENNE M. (ed.), 2010, La modélisation d'accompagnement: une démarche participative en appui au développement durable, Versailles, Quæ.

FASSIN D., 1999, "L'anthropologie entre engagement et distanciation: essai de sociologie des recherches en sciences sociales sur le sida en Afrique ", in BECKER C. et al. (eds), Vivre et penser le sida en Afrique, Paris : 41-66.

GHIOTTI S., 2006, «Les territoires de l'eau et la décentralisation. La gouvernance de bassin versant ou les limites d'une évidence ", Développement durable et territoires, dossier 6.

GOFFMAN E., 1974, Les rites d'interaction, Paris, Editions Minuit.

GWP (Global Water Partnership), 2000, "Integrated Water Resources Mana-gement", TAC Bacground paper No. 4, Stockholm, GWP.

HAGBERG S. et OUATTARA F., 2012, "Engaging anthropology for development and social change", Bulletin de l'APAD, 34-36.

LAVIGNE DELVILLE P. et SELLAMNA N.-E., 2000, "Introduction », in LAVIGNE DELVILLE P., SELLAMNA N.-E. et MATHIEU M. (eds.), Les enquêtes participatives en débat: ambition, pratiques et enjeux, Paris : $5-15$. 
LIU M., 1997, Fondements et pratiques de la recherche-action, Paris, L'Harmattan.

MALINOWSKI B., 1989, Les Argonautes du Pacifique occidental, Paris, Gallimard.

MOLLE F., 2012, "La GIRE : anatomie d'un concept », in JULIEN F. (ed), La gestion intégrée des ressources en eau en Afrique subsaharienne, Québec, Presses de l'Université du Québec : 23-53.

OLIVIER DE SARDAN J.-P., 2000, «Le "je" méthodologique. Implication et explicitation dans l'enquête de terrain ", Revue Française de Sociologie, 41(3) : 417-445.

OLIVIER DE SARDAN J.-P., 2001, " Les trois approches en anthropologie du développement ", Revue Tiers Monde, 42(168) : 729-754.

OLIVIER DE SARDAN J.-P., 2004, « La rigueur du qualitatif. L'anthropologie comme science empirique ", Espaces Temps, 84-86 : 38-50.

RIBOT J.C., AGRAWAL A. et LARSON A.M., 2006, "Recentralizing While Decentralizing: How National Governments Reappropriate Forest Resources", World Development, 34(11) : 1864-1886.

VENOT J., TOROU B.M. et DARE W., 2014, " Territorialisation ou spatialisation : les agences et comités locaux de l'eau au Burkina Faso ", L'Espace géographique, 2(43) : 148-163.

VIDAL L., 2011, "Le développement comme laboratoire de l'anthropologie ", Ethnologie française, 41(3) : 483-495.

VINCK D., 2007, Sciences et société. Sociologie du travail scientifique, Paris, A. Colin.

VOINOV A. et BOUSQUET F., 2010, "Modelling with stakeholders", Environmental Modelling et Software, 25 : 1268-1281.

WALASZEK A., 2012, "Pratiques de la gestion intégrée des ressources en eau: centralité et représentativité des acteurs au sein d'un comité local de l'eau dans le sud-ouest du Burkina Faso ", Mémoire Master 2 Géographie, Université Paris Ouest Nanterre.

\section{Niviviv}


William's Daré est sociologue,

Chargé de recherche au CIRAD, UPR GREEN

E-mail : william's.dare@cirad.fr

Jean-Philippe Venot est géographe,

Chargé de recherche à l'IRD, UMR G-EAU,

et au Water Resources Management group, Université de Wageningen

E-mail : jean-philippe.venot@ird.fr 